\section{A hydrophobic sequence motif common to N-hydroxylating enzymes}

The first committed step in the biosynthesis of various bacterial and furigal siderophores (low-molecular-weight ircn chelators that are produced in response to iron deficiency) of the hydroxamate type, such as aerobactin, alcaligin anc ferrichrome, involves $\mathrm{N}$-hydroxylation of a primary amino group. This reac ion is catalyzed at the expense of NADPH by a family of FAD-dependent enzymes. Some of the siderophores act as virulence factors ${ }^{1}$. A similar reaction is carried out by a family of mammalian flavin-containing dimethylaniline monooxygenases. In the latter case, the substrates are tertiary and secondary diet-derived alkylamines and, as such, these enzymes play a role in the degradation of xenobiotics. Deficiency in this enzyme activi y was recognized as the cause of the inheritable 'fish-odor syndrome' (trime thylaminuria) which is characterized t.y an increased excretion of malodorous trimethylamine ${ }^{2}$.

A BLAST sear $h^{3}$ in the NCBI non-redundant database (as of 5 August 1997) and sequence alignment with CLUSTALX (Rei. 4) and MACAW (Ref. 5) of four sideroph ore biosynthetic enzymes from Escherichia coli (aerA; iucD) ${ }^{6.7}$, Pseudomonas aeruginosa (pvdA), Bordetella bronchiseptica (alcA) ${ }^{9}$ and Ustilago mavdis (sid 1$)^{10}$ and about 30 sequences of flaxin-containing mammalian monooxygenases (nine represent ative sequences are shown in Fig. 1) revealed three dominant areas of similarity (Fig. 1a, b and c). As expected, all proteins contained two nucleotide-binding folds; the N-terminal fold was assigned as the FAD and the one towards the centre as the NADP binding site (Fig. $1 a$ and $b$, respectively) ${ }^{11,12}$. The FAD-binding site of the mammalian monooxygenases has the typical fingerprint sequence GXGXXG, whereas the siderophore biosynthetic enzymes (alcA, iucD, pvdA and sid1, see Fig. 1) exhibit an exchange of the last glycine to proline. This quite unusual replacement is unique among FAD-dependent enzymes and it was assumed to be the cause of the weak binding of $F A D$ to lysine

$\mathrm{N}^{6}$-hydroxylase (EC 1.14.13..- ${ }^{12}$. Similarly, alc $A$ and pvdA possess an alanine and sidl a serine instead of the last glycine in the putative NADP-binding site.

The third and new sequence similarity was discovered in the C-terminal part of the proteins (Fig. 1c). The similarity starts with a highly conserved aspartate and is followed by eight hydrophobic amino acids. The core region consists of the sequence L/FATGY and ends with a proline after four variable amino acids. An exception was found in the two ornithine $\mathrm{N}^{5}$-hydroxylases (a) Consensus

alcA

iucD

$\operatorname{pvdA}$

sid1

YHX6_YEAST

FMO1_MOUSE

FMO1-RABIT

FMO2 CAVPO

FMO5_RABIT

FMO1-RAT

FMO1-HUMAN

FMO3_HUMAN

FMO4-HUMAN

FMO5_HUMAN

(b)

Consensus

$\begin{array}{ll}\text { alcA } & 190 \\ \text { iucD } & 182 \\ \text { pvdA } & 208 \\ \text { sid1 } & 282 \\ \text { YHX6_YEAST } & 189 \\ \text { FMO1_MOUSE } & 185 \\ \text { FMO1_RABIT } & 184 \\ \text { FMO2_CAVPO } & 185 \\ \text { FMO5_RABIT } & 185 \\ \text { FMO1_RAT } & 185 \\ \text { FMO1_HUMAN } & 184 \\ \text { FMO3_HUMAN } & 184 \\ \text { FMO4_HUMAN } & 184 \\ \text { FMO5_HUMAN } & 185\end{array}$

(c)

Consensus

alcA
iucD
pvdA
sid1
YHX6_YEAST
FMO1_MOUSE
FMO1_RABIT
FMO2_CAVPO
FMO5_RABIT
FMO1_RAT
FMO1_HUMAN
FMO3_HUMAN
FMO4_HUMAN
FMO5_HUMAN

330
323
345
433
257
321
323
321
321
321
320
320
319
321

330

A $\mathrm{T}$

E S

R F

N I

P I

P I

D I

D I

P I

P I I I V

NI DV V I A T G Y S F Y P F L 337

NADP-binding motif

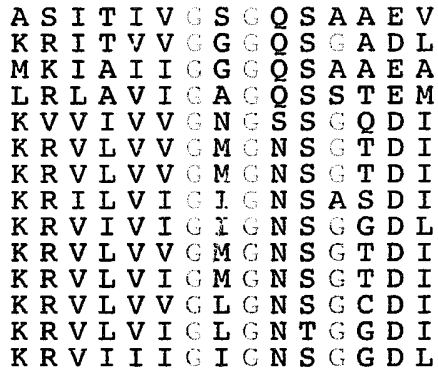

204

196

222

296

203

199
198

199

199

199

198

198

199

p: JC4556

sp: P11295

p: A49892

p: $A 47266$

sp: P38866

sp: P50285

sp: P17636

sp: $P 36366$

sp: Q04799

sp:P36365

sp: Q01740

sp:P31513

sp:P31512

sp:P49326
'FATGY' motif

$\mathrm{XXXATCXXX}$ $\begin{array}{lll}\text { S FA F F L L F } & 338 \\ \text { T F } & 337\end{array}$

G L V L

$\begin{array}{lllll}\text { A V I L A T E R Q L H R Q } & 362 \\ \text { A V F L G T F I R S P S K M } & 450\end{array}$

Y I I

I I V A Y T F F P F L 338

V I V t C T FA F S I 340

VIV $\quad$ TFS F F L 338

VI $A G$ S F F F L 338

S F A F F L 338

I I AVIFA GYS F F P F 336 p: JC4556

sp:P11295

p: A49892

p: $A 47266$

$\mathrm{sp:P38866}$

sp:P50285

sp:P17636

sp: P36366

sp: Q04799

sp: P36365

sp:Q01740

sp:P31513

sp:P31512

sp:P49326

Figure 1

Multiple sequence alignment of $\mathrm{N}$-hydroxylating siderophore biosynthetic enzymes and mammalian $\mathrm{N}$-hydroxylating dimethylaniline monooxygenases. The sequences were aligned using the programs CLUSTALX ${ }^{4}$ and MACAW 5 . (a) FAD-binding motif. (b) NADP-binding motif. (c) 'FATGY' motif. Protein names are given on the left and accession numbers from SWISSPROT (sp) and PIR (p) on the right. The numbers before and after the sequence show the position of the displayed regions in their respective sequences. The first row gives the consensus sequence that is shared by the majority of the sequences. The consensus box is shaded in yellow. Absolutely conserved residues are highlighted in red. Residues where the chemical character is conserved are highlighted in blue. The green shaded area illustrates the exchanged glycine to proline in the FAD-binding motif in the siderophors biosynthetic enzymes. Aligned sequences: alcA, alcaligin biosyntihesis enzyme (Bordetella bronchiceptica); iucD, L-lysine $\mathrm{N}^{6}$-hydroxylase (Escherichia coll); pvdA, L-ornithine $\mathrm{N}^{5}$-oxygenase (Pseudomonas aeruginosa); sid1, L-ornithine $\mathrm{N}^{5}$-oxygenase (Ustilago maydis); YHX6_YEAST, hypothetical $42.4 \mathrm{kDa}$ protein (Saccharomyces cerevisiae); FM01_MOUSE, hepatic flavin-containing monooxygenase 1 (FMO 1) (Mus musculus) (mouse); FM01_RABIT, hepatic flavin-containing monooxygenase 1 (FMO 1) (Oryctolagus cuniculus) (rabbit); FM02_CAVPO, pulmonary flavincontaining monooxygenase 2 (FMO 2) (Cavia porcellus) (guinea pig); FMO5_RABIT, hepatic flavin-containing monooxygenase 5 (FMO 5) (Oryctolagus cuniculus) (rabbit); FM01_RAT, hepatic flavin-containirig monooxygenase 1 (FMO 1) (Rattus norvegicus) (rat); FM01_HUMAN, fetal hepatic flavin-containing monooxygenase 1 (FMO 1) (human); FMO3_HUMAN, hepatic flavin-containing monooxygenase 3 (FMO 3) (human); FM04_HUMAN, hepatic flavin-containing monooxygenase 4 (FMO 4) (human); FMO5_HUMAN, hepatic flavin-containing monooxygenase 5 (FMO 5) (human).

Konstanzer Online-Publikations-System (KOPS) 
(Fig. 1c; pvdA and sid1) where this proline is not conserved. Although some variance is observed, exchanges are conservative in nature, that is, serine to threonine or isoleucine to valine. This $\mathrm{D}(\mathrm{X})_{3}(\mathrm{~L} / \mathrm{F}) \mathrm{ATGY}(\mathrm{X})_{4} \mathrm{P}$-motif was found to be rather hydrophobic in the mammalian monooxygenases ${ }^{11}$, and using the procediure of Kyte and Doolittle ${ }^{1 / 3}$ (a window size of 15 amino acids) this was also confirmed for the siderophore biosynthetic enzymes. In

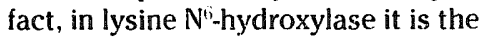
region of the highest hydrophobicity.

A pattern scan using the expasy server (http://expasy.hcuge.ch/sprot/scnpsit2.html) in SWISS-PROT and TREMBL databases showed that the $D(X)_{3}(L / F) A T G Y(X)_{4} P$, $\mathrm{D}(\mathrm{X})_{33}(\mathrm{~L} / \mathrm{F})$ ATGY or (L/F)ATGY $(\mathrm{X}), \mathrm{P}$ motif is only present in: mammalian flavin-containing dimethylaniline monooxygenases; L-lysine $\mathrm{N}^{\mathrm{b}}$-hydroxylase (Escherichia coli); L-ornithine $\mathrm{N}^{5}$-oxygenase (Pseudomonas aeruginosa); alcaligen biosynthesis enzyme; and a hypothetical $42.4 \mathrm{kDa}$ yeast enzyme, which, according to a BLAST search and CLUSTALX sequence alignment, is similar to mammalian dimethylaniline monooxygenases. A search using the sequence 'FATGY' alone shows that this pentamer occurs in nearly 25 additional proteins, which are not related to flavin-containing N-hydroxylating enzymes, for example, many DNA-binding proteins like zinc-finger proteins and viral DNA polymerases.
Based on the discovery of the $\mathrm{D}(\mathrm{X})_{3}(\mathrm{~L} / \mathrm{F}) \mathrm{ATGY}(\mathrm{X})_{4} \mathrm{P}$-motif in $\mathrm{N}$-hydroxylating enzymes (Fig. 1c), we propose that this motif is part of a substrate-binding site with the L/FATGY core providing a hydrophobic pocket. The highly conserved aspartate, on the other hand, could serve as a proton-abstracting base to render the amino group more reactive in the reaction with the hydroxylating flavin species, that is, the flavin-4a-hydroperoxide ${ }^{12}$.

\section{References}

1 Weinberg, E. D. (1984) Physiol. Rev. 64. 65-102

2 Mitchell, S. C. (1996) Perspect. Biol. Med. 39. 514-526

3 Altschul. S. F. et al. (1990) J. Mol. Biol. 215. 403-410

4 Thompson. J. D.. Higgins, D. G. and Gibson, T. J. (1994) Nucleic Acids Res. 22. 4673-4680

5 Schuier. G. D., Altschul, S. F. and Lipman. D. J. (1991) Proteins 9. 180-190

6 De Lorenzo. V. Bindereif. A.. Paw. B. H. and Neilands, J. B. (1936) J. Bacteriol 165. 570-578

7 Gross. R.. Engelbrecht, F. and Braun. V. (1984) Mol. Gen. Genet. 196, $7 i_{\mathrm{r}}-80$

8 Visca. P., Ciervo, A. and Orsi. N. (1994) J. Bacteriol 176, 1128-1140

9 Kang, H. Y.. Brickman, T. J.. Beaumont. F. C. and Armstrong. S. K. (1996) J. Bacteriol. 178. 4877-4884

10 Mei. B., Budde, A. D. and Leong, S. A. (1993) Proc. Natl. Acad. Sci. U. S. A. 90. 903-907

11 Lomri. N.. Gu. Q. and Cashman. J. R. (1992) Proc. Natl. Acad. Sci. U. S. A. 89. 1685-1689 12 Macheroux. P. Plattner, H. J., Romaguera. A. and Diekmann H. (1993) Eur. J. Biochem. 213. 995-1002

13 Kyte, J. and Doolittle. R. F. (1982) J. Mol. Biol. $157,105-132$

\section{Note added in proof}

A recently cloned and characterized L-ornithine $\mathrm{N}^{5}-\mathrm{oxyg}$ enase from Burkholderia cepacia (accession number: AF013993) also contains the unusual putative $F A D$ binding site 'GXGXXP' (Fig. la) and a 'DXXXLATGY'-motif (Fig. lc) as described here for the hydroxylases.

\section{MATTHIAS STEHR AND HANS DIEKMANN}

Institut für Mikrobiologie, Universität Hannover. Schneiderberg 50, D-30167 Hannover, Germany.

\section{LILIANA SMAU, OLIYER SETH AND SANDRO GHISLA}

Fakultät für Biologie, Universität Konstanz. D-78567 Konstanz. Germany.

\section{MAHAVIR SINGH}

Gesellschaf, für Biotechnologische Forschung $\mathrm{mbH}$, Mascheroder Weg 1. D-38124 Braunschweig, Germany.

\section{PETER MACHEROUX}

Institut für Pflanzenwissenschaften, ETH-Zürich, Universitätstr. 2, CH-8092 Zürich, Svitzerland.

Email: peter.macheroux@ipw.biol.ethz.ch 